\title{
EVALUATING PARASITE DENSITIES AND ESTIMATION OF PARAMETERS IN TRANSMISSION SYSTEMS
}

\author{
HEINZMANN D.* \& TORGERSON P.R.*
}

\section{Summary:}

Mathematical modelling of parasite transmission systems can provide useful information about host parasite interactions and biology and parasite population dynamics. In addition good predictive models may assist in designing control programmes to reduce the burden of human and animal disease. Model building is only the first part of the process. These models then need to be confronted with data to obtain parameter estimates and the accuracy of these estimates has to be evaluated. Estimation of parasite densities is central to this. Parasite density estimates can include the proportion of hosts infected with parasites (prevalence) or estimates of the parasite biomass within the host population (abundance or intensity estimates). Parasite density estimation is often complicated by highly aggregated distributions of parasites within the hosts. This causes additional challenges when calculating transmission parameters. Using Echinococcus spp. as a model organism, this manuscript gives a brief overview of the types of descriptors of parasite densities, how to estimate them and on the use of these estimates in a transmission model.

KEY WORDS : mathematical modelling, two part conditional model, Echinococcus, negative binomial distribution, aggregation.

T There are a number of ways to describe the parasite density in the population and the estimation of parameters in the transmission systems. The parameters that are being estimated may also depend upon the method of estimating the parasite density within the population of interest. In addition there may be inherent uncertainty because of errors in the methods or the diagnostic tests used to estimate the parasite densities.

Parasite prevalence is the simplest measure of parasite density and often the easiest to estimate. It is simply the proportion of animals harbouring the parasite in the population. However, it is only a qualitative measure and hence may not be representative of the parasite biomass in the system. Parasite abundance is the mean number of parasites per host and gives an indication of the total biomass of the parasite population

* Institute of Parasitology, University of Zurich, Winterthurestrasse 266a, 8057 Zurich, and Institute of Mathematics, University of Zurich, Winterthurerstrasse 190, 8057 Zürich.

Correspondence: P.R. Torgerson, Insitute of Parasitology, University of Zurich, Winterthurestrasse 266a, CH-8057 Zurich.

Tel.: + 41446358535 - Fax: + 41446358907 .

E-mail: paul.torgerson@access.uzh.ch and hence is potentially of greater use in transmission studies. Parasite intensity is the mean number of parasites per infected host and is also often used to estimate total parasite biomass.

Broadly, transmission models can be prevalence-based and density-based. Prevalence models for transmission dynamics divide the host population into a series of compartments containing for example susceptible, infectious and immune (recovered) animals (Anderson \& May, 1982). Density-based models try to explore the mechanism which gave rise to the observed density distribution of the parasites. Problems which arise when dealing with the count distribution of parasites in the host population include density-dependent mechanisms acting at various stages of the parasite life cycle, e.g. worm fecundity, survival and establishment. These mechanisms depend critically on the distribution of worms between hosts (Anderson \& Gordon, 1982; Medley, 1992).

The primary role of both types of models is to represent transmission systems and unexplained variability (Cox, 2007). Ideally accurate data should be used to estimate the model parameters. However, there may be inherent uncertainty because of errors in the methods used to estimate the parasite densities. Appropriate mathematical models should be used that give a natural description of the underlying distribution of the observed data. This manuscript gives a brief overview of the types of descriptors of parasite densities, how to estimate them and then on the use of these estimates in the transmission systems. Throughout we will use Echinococcus spp. to illustrate how parasite densities can be estimated and how they can be used in a simple transmission model.

\section{METHODS OF MEASURING PARASITE DENSITY}

\section{ABUNDANCE AND INTENSITY}

The most accurate way and often the only way in measuring endoparasite abundance and intensity in hosts is through necropsy studies. How- 
ever the inherent disadvantage is that necropsy studies require the death of the study animal and hence acts as a constraint in modelling parasite biomass, especially in longitudinal studies. With E. granulosus and E. multilocularis accurate counts of adult parasites can be obtain by the intestinal sedimentation and counting techniques following necropsy of the canid definitive host (Eckert, 2003). For other species of parasite, exact enumeration depends on the organ system affected and the parasite in question and there are numerous techniques. For larval echinococcosis it requires careful dissection of the liver, lungs and other organs of affected animals such as sheep or goats (Torgerson et al., 1998). For the liver fluke Fasciola hepatica, for example, quantitative burdens of adult parasites can be obtained by careful dissection of bile ducts and expression of fluke from the liver tissue (Clery et al., 1996). There are fewer opportunities for estimating endoparasite parasite biomass directly in the live animal and these often suffer from low sensitivity. Arecoline purgation of dogs is one technique to quantitatively estimate burdens in infected dogs (Torgerson et al., 2003a) but the sensitivity could be less than $50 \%$ (Ziadinov et al., 2008). Ultrasound has also been used to estimate larval biomass in infected sheep (Lahmar et al., 2007).

In contrast, ecotoparasite biomass can be estimated more easily such as directly evaluating the numbers of parasites on the skin or counting the parasites on a representative proportion of the animals' total surface area. For example, total tick numbers on horses have been evaluated by collecting ticks from one body side of horses (Bourges et al., 2000; Rüegg et al., 2007). Indirect indicators of parasite biomass could be determined by measuring densities of free living stages such as eggs in the faeces of infected animals. Quantitative faecal egg counts can differentiate between heavily or less heavily infected animals. The problem in making conclusions about the parasite density within the host is that there are a number of variables that affect faecal egg counts independently of the parasite biomass within the infected host. These include the faecal consistency and the diet of the infected animal. There are also density dependent constraints on worm fecundity with increasing worm burdens resulting in fewer eggs per worm produced (Anderson \& Schad, 1985). Nevertheless, faecal egg counts have been shown to be a reliable indicator of host parasite burden in some systems such as an estimate of Trichostrongylus tenuis levels in the red grouse (Seivwright et al., 2004). Arguably, in transmission studies quantifying the number of infectious stages produced by an infected animal is likely to be of greater significance than the actual numbers of endoparasites in the animal itself.

\section{PREVAlENCE}

Measuring prevalence is more straightforward than quantitative evaluation of parasite burdens in hosts. It is often relatively undemanding to demonstrate the presence of a parasite in a particular host, although demonstrating its absence may be more of a challenge. A variety of diagnostic techniques can be used and for gastrointestinal parasites coproscopy is often a method of choice. Thus the detection of specific eggs or larval forms of helminth parasites in the host faeces can be used to confirm the parasite is present. A number of parasites however have similar eggs. These include the trichostrongyle parasite in ruminants or taeniid parasites of canids. Trichostrongyle eggs can be differentiated by faecal culture and identification of the larva (van Wyk et al., 2004). PCR techniques can also be employed for a variety of systems such as gastrointestinal nematodes of cattle (Zarlenga et al., 2001) or taeniid eggs observed in dog faeces (Trachsel et al., 2007). For certain parasites such as Schistosoma haematobium urine examination can be used to detect eggs. Many other techniques have been developed to indicate the presence of parasite infections. For gastrointestinal parasites for example, copro-antigen techniques have been developed for the detection of Echinococcus spp. (Allan et al., 1992; Deplazes et al., 2003; Deplazes et al., 1992) and for fasciolosis (Mezo et al., 2004). Serological tests are widespread for the detection of veterinary and human parasites and direct blood smears can detect a variety of protozoa and other parasites.

\section{DIAGNOSTIC UNCERTAINTY}

$\mathrm{E}$ ven simple evaluation of the presence or absence of a parasite in a host is subject to errors which can depend on the diagnostic procedure used to identify the parasite's presence. Direct visualisation and morphological confirmation of parasite eggs or the parasites themselves normally confirms the presence of the parasite in the host. Likewise highly specific PCR tests can generally confirm parasite infection. However, diagnostic tests such as coproantigen detection or serology usually have a specificity of less than $100 \%$ so a positive reaction in an individual animal does not absolutely prove infection. As the sensitivity of any test is seldom if ever $100 \%$ a negative test does not rule out the presence of infection.

Despite these shortcomings, if knowledge of the test performance is known then in population and transmission studies the observed test results can be adjusted to account for the imperfect sensitivity and specificity of the detection system used, at least in theory. However, the diagnostic parameters of a particular test may vary according to the population in which the test is used (Leeflang \& Bossuyt, 2005) and hence even prior information on test performance may prove to 
be inaccurate for the particular population in question. Ways to circumvent these problems are becoming available. For example, the use of two or more test systems when assessing the prevalence of parasite infection in different groups of animals for transmission studies can be utilised, particularly if there is minimal dependence between the tests and/or one of the test has $100 \%$ specificity (such as PCR or visualisation of the actual parasite or its eggs). The Hui Walter model (Hui \& Walter, 1980; Toft et al., 2005) for using multiple diagnostic techniques in the absence of a gold standard can then be incorporated into the transmission model, using for example Bayesian techniques to estimate true prevalences and transmission parameters rather than ones which are based on test prevalences. This is illustrated in a recent manuscript on the transmission of echinococcosis in dogs in Kyrgyzstan (Ziadinov et al., 2008).

\section{CHARACTERISTICS OF EMPIRICAL DATA AND MODEL CHOICE}

$\mathrm{P}$ arasite abundance data is usually aggregated within a population of hosts. For example, adult E. gramulosus counts in samples from South Kazakhstan (Torgersonet al., 2003a), from Tunisia (Lahmar et al., 2001) and from China (Budke et al., 2005) indicate a large proportion of definitive hosts free of infection, namely $76.9 \%, 72.9 \%$ and $91.6 \%$ respectively. The remaining non-zero loads are aggregated, i.e. most of the parasite biomass being in a relatively small proportion of individuals. There are individual animals in the sample carrying much more than 10,000 parasites. In the intermediate host sheep, a similar pattern can be observed. Hydatid cyst counts in sheep in samples from Kazakhstan (Torgerson et al., 2003b) and Jordan (Torgersonet al., 1998) shows a similar albeit less extreme pattern of aggregation. Many sheep are free of E. granulosus cysts, whilst others have individual burdens of up to 50-80 cysts.

The distribution of parasites and cysts in both, the dogs and the sheep, implies that it is often useful to describe parasite populations not only in terms of their mean abundance or intensity, but also the degree of aggregation within the host. Models used in such settings may be purely descriptive representations of the observed pattern. An example is the negative binomial distribution which is a mixture of Poisson distributions and allows, compare to the standard Poisson distribution, a more flexible estimation, especially when aggregation is present. Alternatively, models used to explain such observed patterns may tackle the generation mechanism which has produced the measured data. Such models in general have parameters which can directly be interpreted in a biological context, which is not the case for the parameters of the negative binomial distribution. Models dealing with the generation mechanism are especially useful when the goal is to estimate the transmission pressure or even rate of loss of infections. Such parameters can be incorporated into a (data) generating mechanism and hence can be estimated based on the data and yields a straight-forward interpretation. The estimated parameters are directly related to a mechanism in the model.

We will illustrate such mechanism-based models for estimating prevalence and density patterns of Echinococcus spp.

\section{MODELLING AGGREGATED COUNT DATA WITH AN EXCESS OF ZEROS}

The previous section has indicated that the natural abundance of E. granulosus in dogs and sheep exhibits two well-known features. A substantial proportion of zeros and the remaining nonzero loads are positively skewed. To model such kind of data, different approaches can be used. We will focus here on the two-part conditional model (TPC). Let $X$ be a random variable which models the burden in animals which has mixture distribution

$$
\begin{aligned}
& P(X=0)=1-q \\
& P(X=x)=q f(x), x=1,2, \ldots
\end{aligned}
$$

where $q$ is the probability of observing a nonzero load and $f$ is a zero-truncated probability mass function. The TPC (1) separates processes that determine whether or not parasites in the intermediate or definitive host are present from the processes determining the number of parasites, given they are present. Formulating the model in a two-part conditional frame ensures that $q$ (which can be seen as the prevalence) and the parameters of the zero-truncated distribution $f$ can be estimated separately (Welsh et al., 1996), i.e. the model fitting and model interpretation of the components can be done independently.

For our application of E. granulosus, the infection, and thus the development of parasites in dogs, is based on the ingestion of infective cysts from a dead sheep. Hence a two part infection mechanism for dogs can be supposed, one for the contact with an infective (dead) sheep, i.e. a sheep harbouring infective cysts, and a second for the number of parasites acquired conditional on infectivity of the sheep. The contact part is depending on the availability of offal from sheep (or other suitable intermediate hosts) and the feeding behaviour of dogs whereas the second part depends on the number of infective cysts in the dead sheep and on the resistance of the dog to infection once exposed to the parasite. 
Analogously the infection in sheep may well be the result of ingesting occasional egg clumps rather than by separately ingesting single eggs because of the aggregated nature of egg excretion by the definitive host. Hence a two part infection mechanism for sheep is also reasonable, one for the contact and ingestion of egg clumps (from dog faeces), and a second for the number of cysts acquired conditional on infectivity of the clump. Thus the TPC methodology is plausible and will be illustrated by the estimation of the (prevalence) function $q$.

\section{PREVALENCE-BASED TRANSMISSION MODELS}

\section{MODEL SPECIFICATIONS}

T The data samples previously introduced were sampled from cross-sectional studies. Since such studies do not follow-up individuals, a time-dependent model is (almost) impossible to construct from this data. But by assuming that the disease is in an endemic (or steady) state, cross-sectional data stratified according to host age may be interpreted as being measures at different time points. Thus an age-dependent mechanism in the model can describes the time evolution of parasite transmission.

To estimate the prevalence function $q$ given in (1) from those data, let us derive an age-dependent prevalence equation as follows. Assume that the dog population (analogous for sheep) is divided into two states, according to their infection history. Let $S(t)$ be the proportion of susceptible non infected animals at time $t$ and let $q(t)$ be the proportion of infected animals (prevalence) at time $t$, such that $S(t)+q(t)=1$ for each $t>0$. In each time interval of length $\Delta t$, all dogs in the state $S(t)$ are exposed to an infection with rate $\beta \Delta t$, where $\beta$ is the prevailing infection pressure in terms of infections per time unit. In each time interval $\Delta t$, infected dogs in $q(t)$ will loose their infection at a rate of $\mu \Delta t$. The loss of an infection can either be caused by the death of all parasites in an animal or by the death of the host itself in which case it will be replaced by a susceptible but not yet infected newborn. To describe the transition between the two states, the following ordinary differential equation may be used (Roberts et al., 1986, 1987).

$$
\frac{d q(t)}{d t}=-(\beta+\mu) q(t)+\beta
$$

Assuming that there are no established infections at age $O$, i.e. $q(O)=O$, equation (2) can be solved for

$$
q(t)=\frac{\beta}{\beta+\mu}(1-\exp (-(\beta+\mu) t)) .
$$

The asymptotic prevalence of model (3) is $q(\infty)=$ $\beta /(\beta+\mu)$, i.e. $q(\infty)$ is the upper limit for the prevalence for increasing time. The age-dependent prevalence equation (3) can be modified in order to test different hypotheses about the generation mechanism. We will consider two such possible modifications. To test the hypothesis that the loss rate of infection $\mu$ is zero, i.e. that life-long persistence of an infection may be assumed, equation (3) can be re-written as

$$
q(t)=1-\exp (-\beta t)
$$

with corresponding asymptotic prevalence $q(\infty)=1$. The second modification of model (3) is to assume an age-dependent infection rate which may explain a certain behavioural change in older animals or an acquired immunity with age. Take $\beta=\beta_{0} \exp (-\gamma t)$, where $\beta_{0}$ and $\gamma$ are constant and $t$ indicates the age, replace this expression into the ordinary differential equation (2) and solve with the same initial conditions as before results in (after some algebraic transformations)

$q(t)=\frac{\int_{0}^{t} \beta \exp \left(-\gamma s+\frac{\beta}{\gamma}(1-\exp (-\gamma s))+\mu s\right) d s}{v(t)}$,

where

$$
v(t)=\exp \left(\frac{\beta}{\gamma}(1-\exp (-\gamma t))+\mu t\right)
$$

Other possibilities would be to introduce an age-shift $t_{0}$ to verify if ages are systematically over (or under) estimated or if there are no established infections in young animals. Thus we have an equation of the form

$$
q(t)=\frac{\beta}{\beta+\mu}\left(1-\exp \left(-(\beta+\mu)\left(t-t_{0}\right)\right),\right.
$$

where ages $<t_{0}$ will be truncated at zero. Since agerecords may be inaccurate the methodology of errorin-variables (Cook \& Stefanski, 1994) can be applied.

\section{Parameter ESTIMATION AND CONFIDENCE SPACE}

Based on the results of a likelihood-ratio test of the model (3) versus (5) and (6) in Table I, it turns out that both modified prevalence equations do not statistically significant improve the fit and hence the ini-

\begin{tabular}{lcccc}
\hline Model & LL & LRT & df & p-value \\
\hline$(3)$ & -325.206 & - & - & - \\
$(5)$ & -324.055 & 2.302 & 1 & 0.129 \\
$(6)$ & -324.583 & 1.246 & 1 & 0.264 \\
\hline
\end{tabular}

Table I. - The likelihood value (LL), the likelihood ratio test statistics (LRT), the degree of freedom (df) and the resulting p-value for the comparison of model (2) to model (4) and (5) for the dog sample from Kazakhstan. 


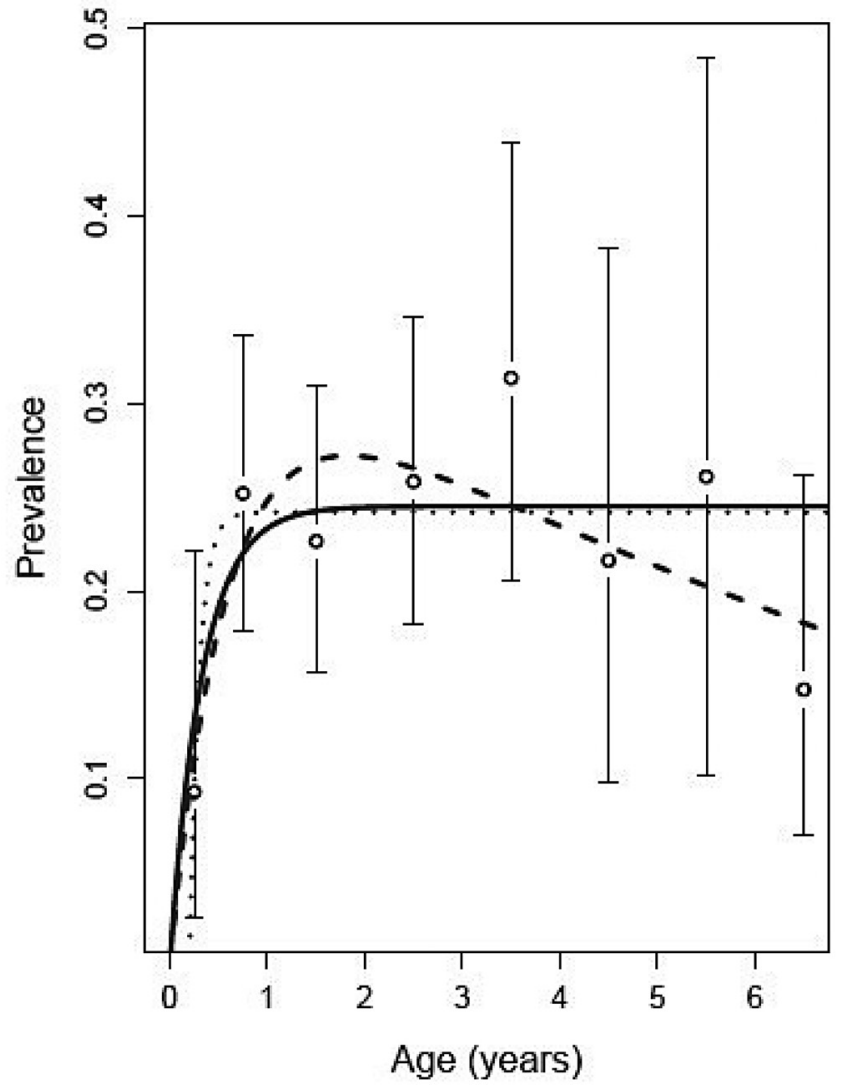

Fig. 1. - Maximum likelihood fit of the prevalence in the dog sample from Kazakhstan of model (3) (solid line), model (5) (dashed line) and model (6) (dotted line) are plotted against the observed prevalences (points) and their corresponding $95 \%$ confidence intervals (vertical bars). Model (4) is omitted here since empirical data in all dog sample does not support an asymptotic prevalence of 1 .

tial prevalence equation (3) provides the best fit to the data. The corresponding prevalence curves for all models are represented in Figure 1. The application of the errors-in-variables method (not shown here) has not significantly improved the fit. The estimates of model (3) for the prevailing infection pressure and the loss rate of infection are 0.744 and 2.291 in farm dogs in Kazakhstan. These indicate, at the time of the study, that the mean time to exposure to infection for a dog in the study population is $1 / 0.744=1.344$ years, and the mean survival time for an infection is $1 / 2.291=$ 0.436 years. In this case the TPC model gives a lower estimate of parasite survival time than that reported using an alternative transmission model (Torgersonet al., 2003a). However, a simultaneous two-dimensional confidence region for the parameter couple is displayed in Figure 2, indicating that the estimation of the couple provides a huge uncertainty and that it is almost impossible to adequately estimate both parameters at the same time. An alternative is provided by fixing one of the parameters, for example $\mu$, and then to estimate the remaining parameter. This example shows that

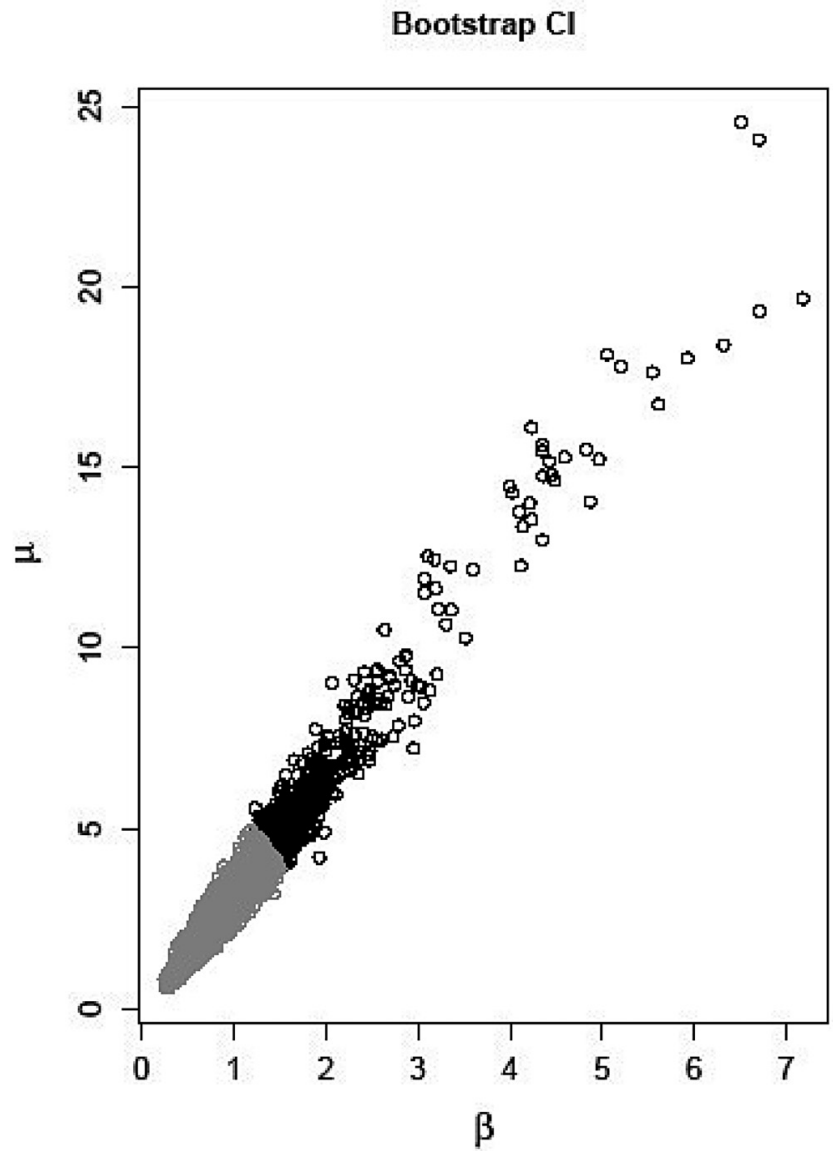

Fig. 2. - Plot of estimates of the parameters couple $(\beta, \mu)$ for 1,000 bootstrap samples, resampled from the original data with replacement. The grey area corresponds to the computed two-dimensional $95 \%$ bootstrap confidence region.

parameter estimation always incorporates some uncertainty which may make it difficult or even impossible to adequately estimate all parameters simultaneously. Prior knowledge or a Bayesian approach can be used to overcome the problem. For example data from experimental infections could be used as a prior estimate of the survival time (Kapel et al., 2006).

\section{CONCLUSIONS}

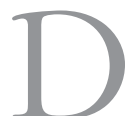
ensity measures of parasites in hosts is a central issue in the estimation of parameters of models describing the transmission systems of parasitic diseases. Based on incomplete or inaccurate data, the statistical analysis involves some kind of uncertainty sources which needs to be accounted for in order to make inference of the results. The choice of model will, by its nature, affect the interpretation of the results. For example, we illustrated a two-part conditional model which can be used to separate data generation mechanisms in order to obtain a clearer picture 
what is actually (or hypothetical) happening in the transmission system. A detailed description is given for a prevalence-based model which is built on the idea to explain the mechanism which gave rise to the observed prevalence pattern in nature. Such models can outperform purely descriptive methods, such as fitting a negative binomial distribution to data, since latter do not really provide an explanation of the natural data generating process. Thus analytical methods and mathematical models play a major role in helping us to understand complex real-life dynamics of associations between hosts and parasites. They can provide a tool for designing programs of public health and primary health care, for animal disease control and for understanding the host parasite dynamics in wildlife. However, it is important to stress that such models are only descriptions of reality, always relying on some assumptions to simplify real-world phenomena. Hence interpretation of models must be conditional of those underlying assumptions.

\section{ACKNOWLEDGEMENTS}

This work was supported by the Schweizerischer Nationalfonds ref 3152A0-107726/1

\section{REFERENCES}

Allan J.C., Craig P.S., Garcia N.J., Mencos F., Liu D., Wang Y., Wen H., Zhou P., Stringer R. \& Rogan M. Coproantigen detection for immunodiagnosis of echinococcosis and taeniasis in dogs and humans. Parasitology, 1992, 104, 347-356.

ANDERSON R.M. \& GORDON D.M. Processed influencing the distribution of parasite numbers within host populations with special emphasis on parasite-induced host mortalities. Parasitology, 1982, 85, 373-398.

Anderson R.M. \& MAY R.M. Population biology and infectious diseases, in: Report of the Dahlem Workshop on Population biology of infectious disease agents, Springer-Verlag, 1982.

ANDERSON R.M. \& SCHAD G.A. Hookworm burden and faecal egg counts: an analysis of the biological basis of variation. Transactions of the Royal Society of Tropical Medicine E Hygiene, 1985, 79, 812-825.

Bourges L.M.F., Oliveira P.R. \& Ribeiro M.F.B. Seasonal dynamics of Anocentor nitens on horses in Brazil. Veterinary Parasitology, 2000, 89, 165-171.

Budke C.M., Jiamin Q., Craig P.S. \& Torgerson P.R. Modeling the transmission of Echinococcus granulosus and Echinococcus multilocularis in dogs for a high endemic region of the Tibetan plateau. International Journal for Parasitology, 2005, 35, 163-170.

Clery D., Torgerson P. \& Mulcahy G. Immune responses of chronically infected adult cattle to Fasciola hepatica. Veterinary Parasitology, 1996, 62, 71-82.
CoOK J.R. \& Stefanski L.A. Simulation-extrapoloation estimation in parametric measurement erro models. Journal of the American Statistical Association, 1994, 89, 1314-1328.

Cox D.R. Applied statistics: a review. Annals of Applied Statistics, 2007, 1, 1-16.

Deplazes P., Gottstein B., Eckert J., Jenkins D.J., Ewald D. \& Jimenez-Palacios S. Detection of Echinococcus coproantigens by enzyme-linked immunosorbent assay in dogs, dingoes and foxes. Parasitology Research, 1992, 78, 303-308.

Deplazes P., Dinkel A. \& Mathis A. Molecular tools for studies on the transmission biology of Echinococcus multilocularis. Parasitology, 2003, 127, S53-S61.

ECKERT J. Predictive values and quality control of techniques for the diagnosis of Echinococcus multilocularis in definitive hosts. Acta Tropica, 2003, 85, 157-163.

HuI S.L. \& WALTER S.D. Estimating the error rates of diagnostic tests. Biometrics, 1980, 36, 167-171.

Kapel C.M.O., Torgerson P.R., Thompson R.C.A. \& Deplazes P. Reproductive potential of Echinococcus multilocularis in experimentally infected foxes, dogs, raccoon dogs and cats. International Journal for Parasitology, 2006, 36, 79-86.

Lahmar S., Kilani M. \& Torgerson P.R. Frequency distributions of Echinococcus granulosus and other helminths in stray dogs in Tunisia. Annals of Tropical Medicine E Parasitology, 2001, 95, 69-76.

Lahmar S., Ben Chéhida F., Pétavy A.F., Hammou A., lahmar J., Ghannay A., GHarbi H.A. \& SARCiron M.E. Ultrasonic screening for cystic echinococcosis in sheep in Tunisia. Veterinary Parasitology, 2007, 143, 42-49.

LeEflang M.G. \& BossurT P.M.M. test accuracy is likely to vary depending on the population it is based on. Veterinary Parasitology, 2005, 134, 189.

MedLey G.F. Which comes first in host-parasite systems: density dependence or parasite distribution? Parasitology Today, 1992, 8, 321-322.

Mezo M., Gonzalez-Warleta M., Carro C. \& Ubeira F.M. An ultrasensitive capture ELISA for detection of Fasciola bepatica coproantigens in sheep and cattle using a new monoclonal antibody (MM3). Journal of Parasitology, 2004, 90, 845-852.

Roberts M.G., Lawson J.R. \& Gemmell M.A. Population dynamics in echinococcosis and cysticercosis: mathematical model of the life-cycle of Echinococcus granulosus. Parasitology, 1986, 92, 621-641.

Roberts M.G., Lawson J.R. \& Gemmell M.A. Population dynamics in echinococcosis and cysticercosis: mathematical model of the life-cycles of Taenia hydatigena and T. ovis. Parasitology, 1987, 94, 181-197.

Rüegg S.R., Torgerson P., Deplazes P. \& Mathis A. Age-dependent dynamics of Theileria equi and Babesia caballi infections in southwest Mongolia based on IFAT and/or PCR prevalence data from domestic horses and ticks. Parasitology, 2007, 134, 939-947.

Seivwright L.J., Redpath S.M., Mougeot F., Watt L. \& Hudson P.J. Faecal egg counts provide a reliable measure of Trichostrongylus tenuis intensities in free-living red grouse Lagopus lagopus scoticus. Journal of Helminthology, 2004, $78,69-76$. 
Toft N., Jorgensen E. \& HojgaArd S. Diagnosis diagnostic tests: evaluating the assumptions underlying the estimation of sensitivity and specificity in the absence of a gold standard. Preventive Veterinary Medicine, 2005, 45, 139-162.

Torgerson P.R., Williams D.H. \& Abo-Shehada M.N. Modelling the prevalence of Echinococcus and Taenia species in small ruminants of different ages in northern Jordan. Veterinary Parasitology, 1998, 79, 35-51.

Torgerson P.R., Shaikenov B.S., Rysmukhambetova A.T., AbdyBeKova A.M., Usenbayev A.E. \& Bairtursinov K.K. Modelling the transmission dynamics of Echinococcus granulosus in dogs in rural Kazakhstan. Parasitology, 2003a, 126, 417424 .

TOrgerson P.R., SHaikenov B.S., RYsmukhambetova A.T., ABDyBeKova A.M., Usenbayev A.E. \& Bairtursinov K.K. Modelling the transmission dynamics of Echinococcus gramulosus in cattle and sheep in Kazakhstan. Veterinary Parasitology, 2003b, 114, 143-153.

Trachsel D., Deplazes P. \& Mathis A. Identification of eggs of canine taeniids by multiplex PCR. Parastology, 2007, 134, 911-920.

van Wyk J.A., Cabaret J. \& Michael L.M. Morphological identification of nematode larvae of small ruminants and cattle simplified. Veterinary Parasitology, 2004, 119, 277-306.

Welsh A.H., Cunningham R.B., Donnelly C.F. \& Lindenmayer D.B. Modeling the abundace of rare aniamls: statistical models for counts with extra zeros. Ecological Modeling, 1996, 80, 297-308.

Zarlenga D.S., Chute M.B., Gasbarre L.C. \& Boyd P.C. A multiplex PCR assay for differentiating economically important gastrointestinal nematodes of cattle. Veterinary Parasitology, 2001, 97, 199-209.

Ziadinov I., Mathis A., Trachsel D., Rysmukhambetova A.T., Abdyjaparov T.A., Deplazes P. \& Torgerson P.R. Canine echinococcosis in Kyrgyzstan: epidemiology and transmission analysis incorporating diagnostic uncertainty. International Journal for Parasitology, 2008, 38, 1179-1190. 